First publ. in: Lecture notes in computer science , No 2810 / ed. by M.R. Berthold ...

Heidelberg; Berlin: Springer, 2003, pp. 76-87

\title{
Constructing Hierarchical Rule Systems
}

\author{
Thomas R. Gabriel and Michael R. Berthold \\ Data Analysis Research Lab \\ Tripos, Inc., 601 Gateway Blvd., Suite 720 \\ South San Francisco, CA 94080, USA. \\ \{tgabriel, berthold\}@tripos.com
}

\begin{abstract}
Rule systems have failed to attract much interest in large data analysis problems because they tend to be too simplistic to be useful or consist of too many rules for human interpretation. We present a method that constructs a hierarchical rule system, with only a small number of rules at each stage of the hierarchy. Lower levels in this hierarchy focus on outliers or areas of the feature space where only weak evidence for a rule was found in the data. Rules further up, at higher levels of the hierarchy, describe increasingly general and strongly supported aspects of the data. We demonstrate the proposed method's usefulness on several classification benchmark data sets using a fuzzy rule induction process as the underlying learning algorithm. The results demonstrate how the rule hierarchy allows to build much smaller rule systems and how the model - especially at higher levels of the hierarchy-remains interpretable. The presented method can be applied to a variety of local learning systems in a similar fashion.
\end{abstract}

\section{Introduction}

Data sets obtained from real world systems often contain missing, noisy, or simply wrong records. If one attempts to build a rule model for such data sources, the result is either an overfitted and inherently complex model, which is impossible to interpret, or the model ends up being too simplistic, ignoring most of the interesting aspects of the underlying system as well as the outliers. But even this effect of outlier ignorance is often also not desirable since the very example that was excluded from the model may be caused by a rare but still extremely interesting phenomena. The real challenge is therefore to build models that describe all interesting properties of the data while still being interpretable by the human expert.

Extracting rule models from data is not a new area of research. In [9] and [13] algorithms were described that construct hyperrectangles in feature space. The resulting set of rules encapsulates regions in feature space that contain patterns of the same class. Other approaches, which construct fuzzy rules instead of crisp rules, were presented in [1611] and [12]. All of these approaches have in common that they tend to build very complex rule systems for large data sets originating from a complicated underlying system. In addition, high-dimensional feature 
spaces result in complex rules relying on many attributes and increase the number of required rules to cover the solution space even further. An approach that aims to reduce the number of constraints on each rule individually was recently presented in 4]. The generated fuzzy rules only constrain few of the available attributes and hence remain readable even in case of high-dimensional spaces. However, this algorithm also tends to produce many rules for large, complicated data sets.

In this paper we describe a method that attempts to tackle this inherent problem of interpretability in large rule models. We achieve this by constructing a hierarchy of rules with varying degrees of complexity. Early attempts to build model hierarchies have been published previously. In [5] an approach to build unsupervised hierarchical cluster models was described, however, the resulting system of clusters does not offer great interpretability. A method to build hierarchies of rule systems was described in [8. Here, an ensemble of rule sets with different granulation is built at the beginning. Starting with a coarse granulation (usually having only two membership functions for each attribute), the remaining rule sets exhibit increasingly finer granulation. In the resulting multirule table the grade of certainty for each rule is then used for pruning, that is, rules with a low grade of certainty will be removed from the rule set. However, this method still exhibits the complexity problem described above - in case of high-dimensional feature spaces, the resulting number of rules will increase exponentially with the dimensionality of the space. As a result, each rule relies on all attributes, making it very hard to interpret.

The approach presented here builds a rule hierarchy for a given data set. The rules are arranged in a hierarchy of different levels of precision and each rule only depends on few, relevant attributes thus making this approach also feasible for high-dimensional feature spaces. Lower levels of the hierarchy describe regions in input space with low evidence in the given data, whereas rules at higher levels describe more strongly supported concepts of the underlying data. The method is based on the fuzzy rule learning algorithm mentioned above [4], which builds a single layer of rules autonomously. We recursively use the resulting rule system to determine rules of low relevance, which are then used as a filter for the next training phase. The result is a hierarchy of rule systems with the desired properties of simplicity and interpretability on each level of the resulting rule hierarchy. We evaluate the classification performance of the resulting classifierhierarchy using benchmark data sets from the European StatLog-Project 7]. Experimental results demonstrate that fuzzy models at higher hierarchical levels indeed show a dramatic decrease in number of rules while still achieving better or similar generalization performance than the fuzzy rule system generated by the original, non-hierarchical algorithm.

\section{The Underlying Fuzzy Rule Algorithm}

The underlying, non-hierarchical fuzzy rule learning algorithm is described in [4]. The algorithm constructs a set of fuzzy rules from given training data. The 
resulting set of rules can then be used to classify new data. We briefly summarize the used type of fuzzy rules before explaining the main structure of the training algorithm.

\subsection{Fuzzy Rule Systems}

The underlying fuzzy rule systems are based on a local granulation of the input space for each rule, so that rules can be independently interpreted:

$$
\begin{array}{cccccc}
\mathcal{R}_{1}^{1}: \text { IF } x_{1} \text { IS } \mu_{1,1}^{1} & \text { AND } \cdots & \text { AND } x_{n} \text { IS } \mu_{n, 1}^{1} & \text { THEN } & \text { class } 1 \\
\vdots & & & \vdots & & \vdots \\
\mathcal{R}_{r_{1}}^{1}: \text { IF } x_{1} \text { IS } \mu_{1, r_{1}}^{1} & \text { AND } \cdots & \text { AND } x_{n} \text { IS } \mu_{n, r_{1}}^{1} & \text { THEN } & \text { class } 1 \\
\vdots & & & \vdots & & \vdots \\
\mathcal{R}_{r_{k}}^{k}: \text { IF } x_{1} \text { IS } \mu_{1, r_{k}}^{k} & \text { AND } \cdots & \text { AND } x_{n} \text { IS } \mu_{n, r_{k}}^{k} \text { THEN } & \text { class } c
\end{array}
$$

where $\mathcal{R}_{j}^{k}$ represents rule $j$ for class $k$. The rule base contains rules for $c$ classes and $r_{k}$ indicates the number of rules for class $k\left(1 \leq j \leq r_{k}\right.$ and $\left.1 \leq k \leq c\right)$.

The fuzzy sets $\mu_{i, j}^{k}: \mathbb{R} \mapsto[0,1]$ are defined for every feature $i(1 \leq i \leq n)$ and the overall degree of fulfillment of a specific rule for an input pattern $\boldsymbol{x}=$ $\left(x_{1}, \ldots, x_{n}\right)$ can be computed using the minimum-operator as fuzzy-AND:

$$
\mu_{j}^{k}(\boldsymbol{x})=\min _{i=1, \cdots, n}\left\{\mu_{i, j}^{k}\left(x_{i}\right)\right\} .
$$

The combined degree of membership for all rules of class $k$ can be computed using the maximum-operator as fuzzy-OR:

$$
\mu^{k}(\boldsymbol{x})=\max _{j=1, \cdots, r_{k}}\left\{\mu_{j}^{k}(\boldsymbol{x})\right\} .
$$

From these membership values we then derive the predicted class $k_{\text {best }}$ for an input pattern $\boldsymbol{x}$ as:

$$
k_{\text {best }}(\boldsymbol{x})=\arg \max _{k}\left\{\mu^{k}(\boldsymbol{x}) \mid 1 \leq k \leq c\right\} .
$$

The used fuzzy membership functions are trapezoidal and can be described using four parameters $\left\langle a_{i}, b_{i}, c_{i}, d_{i}\right\rangle$, where $a_{i}$ and $d_{i}$ indicate the fuzzy rule's support-, and $b_{i}$ and $c_{i}$ its core-region for each attribute $i$ of the input dimension. The training algorithm shown in the next section usually only constrains few attributes, that is, most support-regions will remain infinite, leaving the rules interpretable even in case of high-dimensional input spaces.

\subsection{Construction of Fuzzy Rules}

The original algorithm [4] will be abbreviated as FRL (Fuzzy Rule Learner) in the following. During the training process all existing example patterns are subsequentially presented to the growing model. The algorithm then introduces new fuzzy rules when necessary and adjusts the coverage (support- and coreregions) of already existing rules in case of conflicts. The complete training data is presented once during each epoch. For each training pattern, three different steps are executed: 
- Cover: If a new training pattern lies inside the support-region of an already existing fuzzy rule of the correct class, its core-region is extended to cover the new pattern. In addition, the weight of this rule is incremented.

- Commit: If the new pattern is not yet covered, a new fuzzy rule belonging to the corresponding class will be created. The new example is assigned to its core-region, whereas the overall rule's support-region is initialized "infinite", that is, the new fuzzy rule is unconstrained and covers the entire domain.

- Shrink: If a new pattern is incorrectly covered by an existing fuzzy rule of conflicting class, this fuzzy rule's support-region will be reduced, so that the conflict with the new pattern is avoided. The underlying heuristic of this step aims to minimize the loss in volume (see [4] for details).

The algorithm usually terminates after only few iterations over the training data. The final set of fuzzy rules can be used to compute a degree of class membership for new input patterns as described in Section 2.1.

\section{$3 \quad$ Learning Hierarchies of Rule Systems}

The fuzzy rule learner described in the previous section has the usual problems when encountering noisy data or outliers. In such cases an excessive number of rules is being introduced simply to model noise and outliers. This is due to the fact that the algorithm aims to generate conflict free rules, that is, examples encountered during training will result in a non-zero degree of membership only for rules of the correct class. Unfortunately, in case of noisy data, which distorts class boundaries, such an approach will result in many superfluous rules introduced simply to model these artifacts.

Using an already existing model we can, however, easily determine these parts of a rule model since they tend to have very low relevance. The main idea behind the hierarchical training algorithm is to use those parts of the rule model with low relevance as a filter for the input data, which in turn is used to create the next layer. This recursive process of data cleansing results in higher levels of the hierarchy, which are built only for examples with strong support in the original data set. For this to work, we obviously need to define the notion of rule relevance or importance more precisely.

\subsection{Measuring Rule Relevance and Filtering Outliers}

To measure a rule's relevance often the weight parameter $\omega^{(\mathcal{R})}$ is used, which represents the number of training patterns covered by rule $\mathcal{R}$. A measure for the importance or relevance of each rule can be derived by simply using the percentage of patterns covered by this rule:

$$
\Phi(\mathcal{R})=\frac{\omega^{(\mathcal{R})}}{|\mathbf{T}|}
$$


where $\mathbf{T}$ indicates the set of all training examples. Other measures which are also used determine the loss of information if rule $\mathcal{R}$ is omitted from all rules $\mathbf{R}$ :

$$
\Phi(\mathcal{R})=I(\mathbf{R})-I(\mathbf{R} \backslash\{\mathcal{R}\})
$$

where $I(\cdot)$ indicates a function measuring the information content of a rule set. Most commonly used are the Gini-index and the fuzzy entropy function (see [10] for details):

- Gini-index:

$$
I_{\text {Gini }}(\mathbf{R})=1-\sum_{k=1}^{c} V\left(\mathbf{R}^{k}\right)^{2}
$$

- Fuzzy entropy:

$$
I_{\text {Entropy }}(\mathbf{R})=-\sum_{k=1}^{c}\left(V\left(\mathcal{R}^{k}\right) \log _{2} V\left(\mathbf{R}^{k}\right)\right)
$$

where $\mathbf{R}=\bigcup_{k=1}^{c} \mathbf{R}^{k}$ and $V\left(\mathbf{R}^{k}\right)$ indicate the volume for all rules of class $k$ $\left(\mathbf{R}^{k}=\left\{\mathcal{R}_{1}^{k}, \ldots, \mathcal{R}_{r_{k}}^{k}\right\}\right)$ :

$$
V\left(\mathbf{R}^{k}\right)=\int_{\boldsymbol{x}} \max _{\mathcal{R}^{k} \in \mathbf{R}^{k}}\left\{\mu^{k}(\boldsymbol{x})\right\} \mathrm{d} \boldsymbol{x} .
$$

In [10] it is shown how this volume can be computed efficiently based on a system of fuzzy rules. More extensive overviews can be found in [2].

The choice of relevance metric is made depending on the nature of the underlying rule generation algorithm, as well as the focus of analysis, i.e. the interpretation of important vs. unimportant or irrelevant data points.

Using any of the above-mentioned measures of (notably subjective) relevance, together with a relevance threshold $\theta_{\text {outlier }}$, we can now extract rules with low relevance from the model:

$$
\mathbf{R}^{\text {outlier }}=\left\{\mathcal{R} \in \mathbf{R} \mid \Phi(\mathcal{R}) \leq \theta_{\text {outlier }}\right\} .
$$

Using this outlier model as a filter for a second training phase will then generate a new fuzzy model, which has fewer rules with higher significance. In effect, the original training data is filtered and only data points, which are not covered by the outlier model, are passed onto the next level for training1. For this a filter-threshold $\theta_{\text {filter }}\left(0 \leq \theta_{\text {filter }} \leq 1\right)$ is used:

$$
\mathbf{T}^{\text {relevant }}=\mathbf{T} \backslash\left\{(\boldsymbol{x}, k) \in \mathbf{T} \mid \exists \mathcal{R} \in \mathbf{R}^{\text {outlier }}: \mu_{\mathcal{R}}(\boldsymbol{x}) \geq \theta_{\text {filter }}\right\} .
$$

This procedure was used in 3] to remove potential outliers from the training data. There, the initial model is used to extract an outlier model, which is subsequentially used to filter the data and generate the final rule model. Figure 1 illustrates the flow of this procedure.

\footnotetext{
${ }^{1}$ Note that this procedure is similar to well-known pruning strategies. In this case, however, the pruned model parts are not discarded but instead used as explicit outlier model.
} 


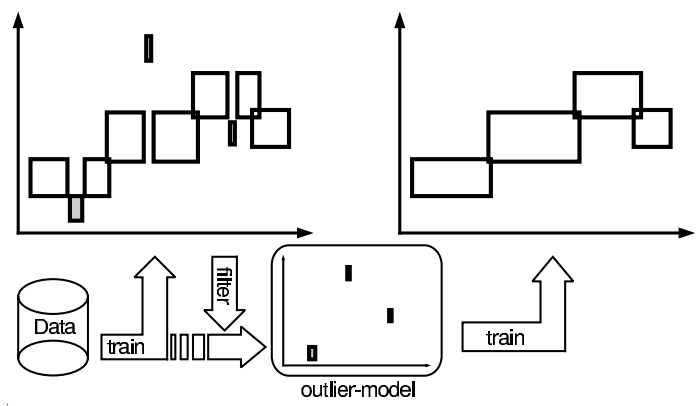

Fig. 1. Two-stage outlier filtering as described in [3].

\subsection{Constructing Rule Hierarchies}

An entire model hierarchy can be obtained by a multi-stage fuzzy rule induction process based on the two-stage procedure described above. This hierarchical training process recursively continues to apply the filtering procedure until a final rule model is generated, where all rules lie above the relevance threshold. The resulting hierarchical Fuzzy Rule Learner (or hFRL) can then formally be described as shown in Table 1 .

After some initializations, line (4) shows the generation of the fuzzy rule model using the original FRL for the input data separately for each level of the hierarchy. From the resulting rule set, we extract rules with low relevance using the outlier-threshold $\theta_{\text {outlier }}$ at line (5). The next step extracts all examples from the rule set using the filter parameter $\theta_{\text {filter }}$. This procedure is repeated until all rules are above the outlier-threshold and the outlier model remains empty. The model hierarchy consists of all outlier models $\mathbf{R}_{i}$ and the rule model of the last iteration, which we denote by $\mathbf{R}_{x}$.

Figure 2 shows a two-dimensional example with patterns of two classes (cross and circle). The rectangles depict the rule's core-regions only. In this example the three-level hierarchy is built using an outlier-threshold $\theta_{\text {outlier }}=2$ and a filterthreshold $\theta_{\text {filter }}=1.0$. That is, rules covering two or less patterns are moved to the outlier model, and only patterns falling inside the core-region of an outlier rule (hence resulting in a degree of membership of at least one) are removed.

Table 1. Algorithm hFRL

\begin{tabular}{ll}
\hline$(1)$ & $\mathbf{T}_{0}=\mathbf{T}$ \\
$(2)$ & $i \leftarrow 0$ \\
$(3)$ & $\operatorname{REPEAT}$ \\
$(4)$ & $\mathbf{R}_{i}=\operatorname{FRL}\left(\mathbf{T}_{i}\right)$ \\
$(5)$ & $\mathbf{R}_{i}^{\text {outlier }}=\left\{\mathcal{R} \in \mathbf{R}_{i} \mid \Phi(\mathcal{R}) \leq \theta_{\text {outlier }}\right\}$ \\
$(6)$ & $\mathbf{T}_{i+1}=\mathbf{T}_{i} \backslash\left\{(\boldsymbol{x}, k) \in \mathbf{T}_{i} \mid \exists \mathcal{R} \in \mathbf{R}_{i}^{\text {outlier }}: \mu_{\mathcal{R}}(\boldsymbol{x}) \geq \theta_{\text {filter }}\right\}$ \\
$(7)$ & $i \leftarrow i+1$ \\
(8) & WHILE $\left|\mathbf{R}_{i-1}^{\text {outlier }}\right|>0$ \\
\hline
\end{tabular}



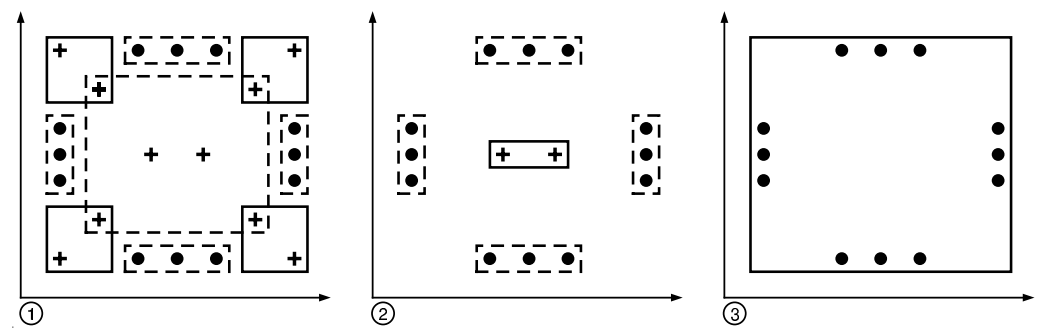

Fig. 2. An example rule hierarchy, using the original rule-based filtering strategy.

Figure 2(1) illustrates the original rule model consisting of nine rules generated at the beginning of the process. The four rules in the corners cover only two patterns each and are therefore added to the first outlier model. The remaining five rules (dashed lines) are not used at this level. The algorithm then removes the corresponding patterns from the set of training data. Figure 2(2) displays the next model, containing five rules. Here, only the rule in the center does not cover more than two examples and composes the second outlier model. The last step generates the final model with one rule covering all remaining patterns of class circle (Figure 2[3)).

The example in Figure 2 shows how this rule-based filtering mechanism can potentially remove large portions of the training data during subsequent stages. This is due to the fact that only individual rule's coverage of patterns is considered. More reasonable results can be achieved using a global, model-based approach.

Our filter criterion can easily be extended accordingly. In addition to the test in line (6), if an outlier rule exists that covers a certain pattern, we now also ensure that no other non-outlier rule covers this pattern:

$$
\mathbf{T}_{i+1}=\mathbf{T}_{i} \backslash\left\{(\boldsymbol{x}, k) \in \mathbf{T}_{i} \mid \ldots \wedge \nexists \mathcal{R} \in \mathbf{R}_{i} \backslash \mathbf{R}_{i}^{\text {outlier }}: \mu_{\mathcal{R}}(\boldsymbol{x}) \geq \theta_{\text {filter }}\right\}
$$

Figure 3 demonstrates the impact of this change using the same data as before. Figure 3(1) again shows the first model in the hierarchy. The rules in the corners are again added to the first outlier model. But this time, the inner data
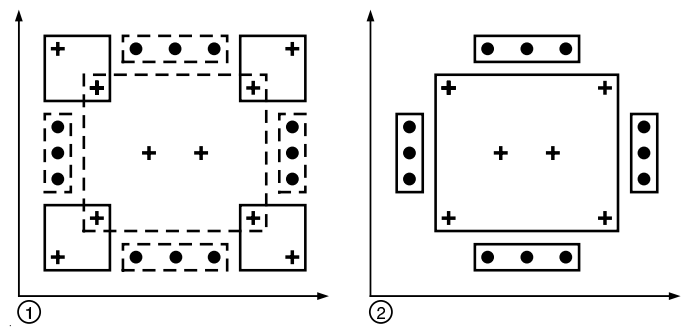

Fig. 3. An example of a model-based rule hierarchy, using the same data as in Figure2 


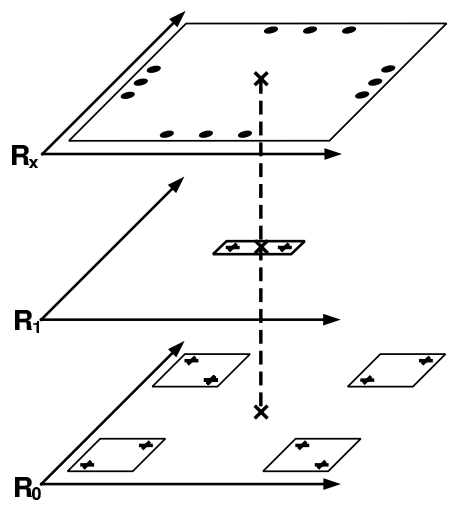

Fig. 4. Classification of a new pattern using a three-level rule hierarchy.

points of these rules are also covered by the rule in the center, which is not part of the outlier model. Therefore these points remain in the training data for the next iteration. The final model in Figure 3(2) contains five rules, all of which are above the outlier-threshold.

This model-based approach enables us to only remove patterns from the next stage that are solely covered by rules of the outlier model and avoids the overly pessimistic problem demonstrated above.

\subsection{Classification Using Hierarchies}

In Section 2.1 we showed how a non-hierarchical rule system can be used for classification of new input patterns, resulting in a set of fuzzy membership values for each class. In case of a hierarchical rule system, the outputs of the rule systems at different levels need to be combined.

Figure 4 shows an example using the hierarchical fuzzy rule model of Figure2, Each rule model, $\mathbf{R}_{0}, \mathbf{R}_{1}$, and $\mathbf{R}_{x}$, provides its own output fuzzy membership degrees for a given input pattern. For the displayed rule system, $\mathbf{R}_{0}$ does not make a prediction for the given example, $\mathbf{R}_{1}$ outputs class cross, and $\mathbf{R}_{x}$ predicts class circle. For our tests we use two classification strategies. One accumulates the fuzzy membership degrees of each level by summing them (FuzzySum $\mathbf{R}_{x} \rightarrow \mathbf{R}_{i}$ ), the other one (FirstHit $\mathbf{R}_{i}\left[\rightarrow \mathbf{R}_{x}\right]$ ), determines the output based on the modellevel that covers the pattern first using a bottom-up strategy.

In the following section we report experimental results using varying depths of the hierarchy. That is, we use the top level model along with an increasing number of outlier models for classification.

\section{Experimental Results}

Eight benchmark data sets from the StatLog-Project 7] are used to demonstrate the performance of the proposed methodology. We follow the division in 


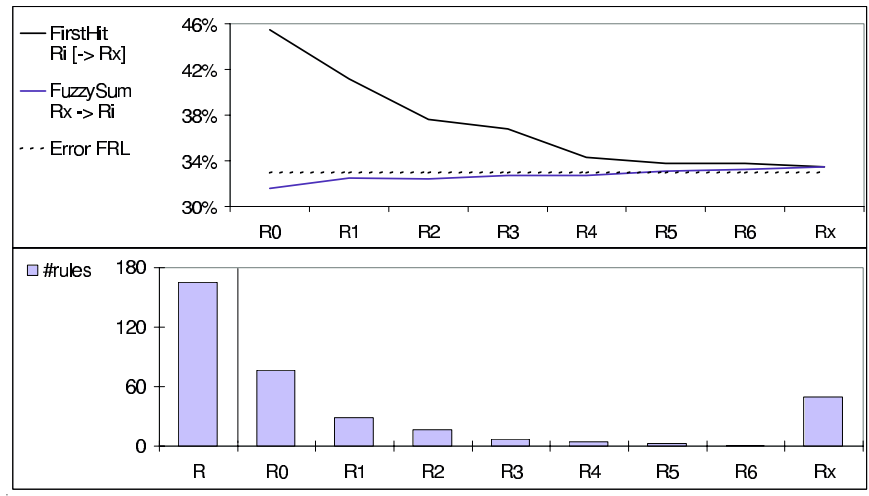

Fig. 5. Vehicle Silhouette data (752 patterns, 8-level hierarchy).

training/test set resp. number of cross validation folds as specified in 7]. For the tests we choose an outlier-threshold $\theta_{\text {outlier }}=5$, that is, rules which cover less than five pattern are moved to the outlier model. We ran preliminary experiments with various choices for this threshold, and this choice seems to be an appropriate value to generate an acceptable depth of the hierarchy for all data sets.

The following paragraphs discuss the results of three of the eight data sets in more detail. In each of the corresponding diagrams, the graph on top shows the error of the non-hierarchical fuzzy rule learner (dashed line), and the performance curves of both classification strategies introduced above. For those two cases, the number of outlier models used for classification was varied (left: using all models down to $\mathbf{R}_{0}$; right: using only the top level model $\mathbf{R}_{x}$ ). In addition, the graph on the bottom shows the number of rules generated by the original fuzzy rule learner $(\mathbf{R})$, as well as the number of rules in each level $\mathbf{R}_{i}$ to $\mathbf{R}_{x}$ of the hierarchy. At the end of this section we summarize the results on these and the remaining four data sets.

\subsection{Vehicle Silhouette Data Set}

Figure 5 illustrates the results on the Vehicle Silhouette data set. The proposed method generates 8 hierarchy levels using the training data. The classical fuzzy model consists of 165 rules producing an error of $33.0 \%$ on the test data. Using only the rule base at the top level $\left(\mathbf{R}_{x}\right)$ a similar performance $(33.5 \%)$ can be achieved with only 49 rules. Slightly better performance $(32.7 \%)$ results from using the FuzzySum-strategy and models $\mathbf{R}_{x}$ to $\mathbf{R}_{4}$ with 57 rules in total. Subsequentially adding models decreases this error further to $31.6 \%$ with altogether 186 rules. On the other hand, using the FirstHit-strategy and all levels of the generated hierarchy produces substantially worse classification results. However, this error declines quickly, when the lower few levels of hierarchy are ignored. This indicates that these parts of the model hierarchy represent artifacts in the training data that are not representative for the underlying concept. 


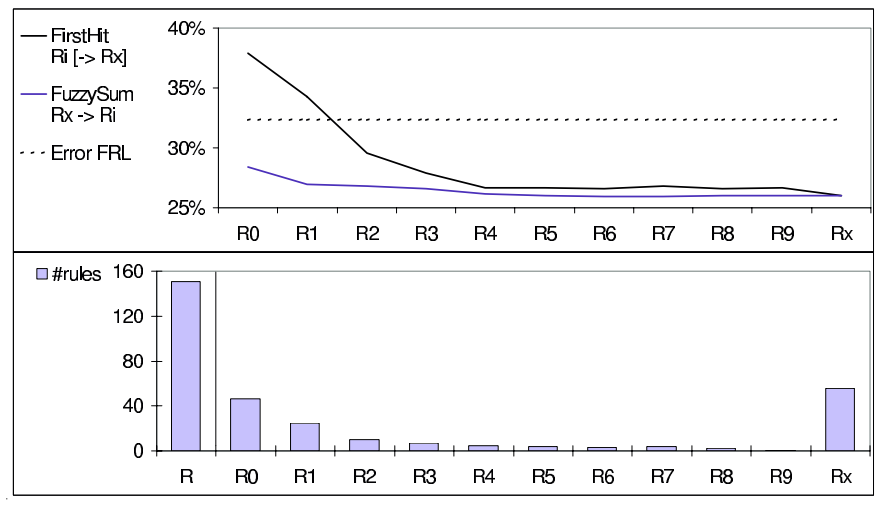

Fig. 6. Pima Indians Diabetes data base (704 patterns, 11-level hierarchy).

\subsection{Pima Indians Diabetes Data Base}

The second test is conducted using the Pima Indians Diabetes data base. Figure 6] shows the results. The non-hierarchical fuzzy model (151 rules) achieves a generalization performance of $32.3 \%$. The hierarchical rule learner generates 11 levels. It is interesting to note that in this case, even model $\mathbf{R}_{x}$ at the top level reaches a substantially better classification performance $(26.0 \%)$ using only 56 rules. The entire hierarchy consists of 162 rules. In this example adding models from lower levels does not increase the performance. But instead, similar to before, including the bottom level harms the performance.

\subsection{Australian Credit Approval}

Experiments on the the Australian Credit Approval with 690 patterns are shown in Figure 7 The non-hierarchical fuzzy rule learning algorithm generates 125 rules with an error of $18.8 \%$ on the test data. The hierarchical rule learner with 5 levels achieves a noticeably better performance of $15.4 \%$ using only 31 rules at the top level. The entire rule hierarchy consists of 91 rules with an minimum error rate of $15.2 \%$. Again, note how when using the FirstHit-strategy the addition of the first outlier model $\mathbf{R}_{0}$ significantly increases the generalization error, indicating a substantial amount of artifacts in the original training data, which were filtered out during the first phase of training.

\subsection{Summary Results}

Table 2 summarizes the results of all eight benchmark data sets from the StatLogProject used for our experiments. The table shows the results in comparison to the non-hierarchical fuzzy rule learner.

The table is separated into three parts. The first column shows the name of the data set, followed by the results of the FRL algorithm (R). Column (2) and (3) list the corresponding number of rules and error rate in percent. The 


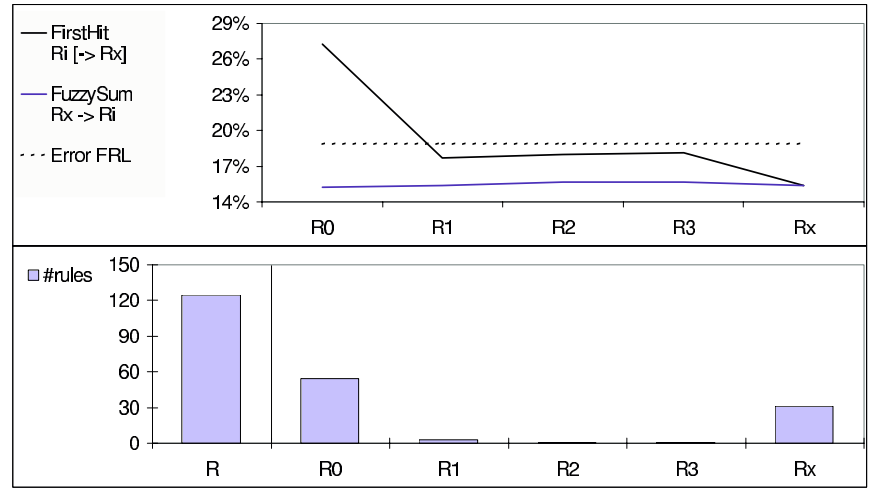

Fig. 7. Australian Credit Approval (690 patterns, 5-level hierarchy).

next column shows the number of generated rule levels, followed by the number of rules and the performance of the top model in the hierarchy $\left(\mathbf{R}_{x}\right)$. Finally, the last two columns show the number of rules and the classification error $\left(\mathbf{R}_{x} \rightarrow \mathbf{R}_{0}\right)$ of the entire hierarchy using the FuzzySum-strategy.

Note how for all eight data sets the number of rules at the top level of the hierarchy is substantially smaller than in the original model. For the Pima Indians Diabetes data base and Australia Credit Approval an even better performance can be achieved using just the top level model. Often even the entire hierarchy is smaller than the classical non-hierarchical rule system (e.g. Australia Credit Approval and Shuttle data set). This clearly indicates that the hierarchical rule learning strategy does indeed extract rules - and subsequentially patterns - with low relevance throughout the training process. The results on the DNA data are not representative since the feature space consists of 240 binary variables. The underlying fuzzy rule learner is highly unstable in this case.

Table 2. Summary of the result for all data sets from the StatLog-Project.

\begin{tabular}{|c|c|c|c|c|c|c|c|}
\hline Data set & $\begin{array}{r}\mathbf{R} \\
\text { \#rules }\end{array}$ & error & \#level & $\begin{array}{r}\mathbf{R}_{x} \\
\text { \#rules }\end{array}$ & error & $\begin{array}{l}\text { FuzzyS } \\
\text { \#rules }\end{array}$ & $\begin{array}{l}\rightarrow \mathbf{R}_{0} \\
\text { error }\end{array}$ \\
\hline Diabetes & 151 & 32.3 & 11 & 56 & 26.0 & 162 & 28.4 \\
\hline Aust. Cred. & 125 & 18.8 & 5 & 31 & 15.4 & 91 & 15.2 \\
\hline Vehicle & 165 & 33.0 & 8 & 49 & 33.5 & 186 & 31.6 \\
\hline Segment & 96 & 3.9 & 6 & 70 & 4.9 & 96 & 4.5 \\
\hline Shuttle & 60 & 0.06 & 3 & 30 & 0.15 & 57 & 0.09 \\
\hline SatImage & 416 & 13.8 & 14 & 132 & 14.0 & 490 & 13.2 \\
\hline DNA & 742 & 32.7 & 9 & 158 & 44.9 & 850 & 39.6 \\
\hline Letter & 2369 & 14.4 & 13 & 714 & 23.0 & 3252 & 14.8 \\
\hline
\end{tabular}




\section{Conclusion}

We have described a method that allows to build a hierarchy of rules based on an underlying, non-hierarchical rule learning algorithm. Using several benchmark data sets we showed how the classification accuracy was comparable and sometimes even better than the underlying, non-hierarchical algorithm and how the resulting rule systems are of substantially reduced complexity. Due to the general nature of the method proposed here, it is possible to apply it to other local learning methods. As long as it is possible to extract parts of the model with low relevance, those pieces can then be used as a filter for subsequent levels in the hierarchy 4 .

\section{References}

1. S. Abe and M.-S. Lan. A method for fuzzy rules extraction directly from numerical data and its application to pattern classifiction. IEEE Transactions on Fuzzy Systems, 3(1):18-28, 1995.

2. C. Apte, S. Hong, J. Hosking, J. Lepre, E. Pednault, and B. K. Rosen. Decomposition of heterogeneous classification problems. Intelligent Data Analysis, 2(2):17-28, 1998.

3. M. R. Berthold. Learning fuzzy models and potential outliers. In Computational Intelligence in Data Mining, pages 111-126. Springer-Verlag, 2000.

4. M. R. Berthold. Mixed fuzzy rule formation. International Journal of Approximate Reasoning (IJAR), 32:67-84, 2003.

5. A. B. Geva. Hierarchical unsupervised fuzzy clustering. IEEE Transactions on Fuzzy Systems, 7(6):723-733, Dec. 1999.

6. C. M. Higgins and R. M. Goodman. Learning fuzzy rule-based neural networks for control. In Advances in Neural Information Processing Systems, 5, pages 350-357, California, 1993. Morgan Kaufmann.

7. D. Michie, D. J. Spiegelhalter, and C. C. Taylor, editors. Machine Learning, Neural and Statistical Classification. Ellis Horwood Limited, 1994.

8. K. Nozaki, H. Ishibuchi, and H. Tanaka. Adaptive fuzzy rule-based classification systems. IEEE Transactions on Fuzzy Systems, 4(3):238-250, 1996.

9. S. Salzberg. A nearest hyperrectangle learning method. In Machine Learning, 6, pages 251-276, 1991.

10. R. Silipo and M. R. Berthold. Input features impact on fuzzy decision processes. IEEE Transcation on Systems, Man, and Cybernetics, Part B: Cybernetics, 30(6):821-834, 2000.

11. P. K. Simpson. Fuzzy min-max neural networks - part 1: Classification. IEEE Transactions on Neural Networks, 3(5):776-786, Sept. 1992.

12. L.-X. Wang and J. M. Mendel. Generating fuzzy rules by learning from examples. IEEE Transactions on Systems, Man, and Cybernetics, 22(6):1313-1427, 1992.

13. D. Wettschereck. A hybrid nearest-neighbour and nearest-hyperrectangle learning algorithm. In Proceedings of the European Conference on Machine Learning, pages 323-335, 1994. 